\title{
Silver-Catalyzed Domino Hydroarylation/Cycloisomerization Reactions of 2-Alkynylquinoline-3-carbaldehydes: Access to (Hetero)arylpyranoquinolines
}

\author{
Alexis Bontemps \\ Gaëlle Mariaule \\ Stéphanie Desbène-Finck \\ Philippe Helissey \\ Sylviane Giorgi-Renault \\ Véronique Michelet* \\ Philippe Belmont* \\ Synthesis 2016, 48, 2178.
}

For the final online and print versions, Scheme 1b has been corrected. 